Lightlike Submanifolds of Semi-Riemannian Manifolds and Applications 
Mathematics and Its Applications

Managing Editor:

M. HAZEWINKEL

Centre for Mathematics and Computer Science, Amsterdam, The Netherlands 


\section{Lightlike Submanifolds of Semi-Riemannian \\ Manifolds and}

Applications

by

Krishan L. Duggal

University of Windsor,

Windsor, Ontario, Canada

and

Aurel Bejancu

Polytechnic Institute of Iaşi,

Iaşi, Romania

SPRINGER-SCIENCE+BUSINESS MEDIA, B.V. 
A C.I.P. Catalogue record for this book is available from the Library of Congress.

ISBN 978-90-481-4678-9

ISBN 978-94-017-2089-2 (eBook)

DOI 10.1007/978-94-017-2089-2

Printed on acid-free paper

All Rights Reserved

(C) 1996 Springer Science+Business Media Dordrecht

Originally published by Kluwer Academic Publishers in 1996

No part of the material protected by this copyright notice may be reproduced or utilized in any form or by any means, electronic or mechanical, including photocopying, recording or by any information storage and retrieval system, without written permission from the copyright owner. 


\section{Table of Contents}

Preface.

\section{Algebraic Preliminaries.}

1.1 Semi-Euclidean spaces

1.2 Subspaces of semi-Euclidean spaces 5

1.3 Linear isometries of semi-Euclidean spaces $\quad 12$

2 Differential-geometric structures on Manifolds.

2.1 Vector bundles

2.2 Differential operators on vector bundles $\quad 23$

2.3 Distributions on manifolds $\quad 32$

2.4 Semi-Riemannian manifolds $\quad 37$

$\begin{array}{ll}2.5 \text { Lightlike manifolds } & 47\end{array}$

3 Geometry of null curves in Lorentz manifolds.

3.1 Frenet frames for a null curve of a Lorentz manifold $\quad 52$

3.2 Null curves of Lorentz manifolds of dimension 4 and $3 \quad 60$

$\begin{array}{ll}3.3 \text { Null curves of } \mathbb{R}_{1}^{m+2} & 68\end{array}$

4 Lightlike hypersurfaces of semi-Riemannian manifolds.

$\begin{array}{ll}4.1 \text { Lightlike transversal vector bundles of a lightlike hypersurface } & 77\end{array}$

4.2 The induced geometrical objects on a lightlike hypersurface $\quad 82$

4.3 The Gauss-Codazzi equations for lightlike hypersurfaces $\quad 93$

4.4 The fundamental theorem for lightlike hypersurfaces 100

4.5 Totally umbilical lightlike hypersurfaces 106

4.6 Lightlike hypersurfaces of semi-Euclidean spaces $\quad 114$

4.7 Lightlike hypersurfaces of $\mathbb{R}_{1}^{3}, \mathbb{R}_{1}^{4}$ and $\mathbb{R}_{2}^{4} \quad 123$

5 Lightlike submanifolds of semi-Riemannian manifolds.

5.1 The transversal vector bundle of a lightlike submanifold $\quad 139$

5.2 The induced geometric objects on lightlike submanifolds $\quad 154$

$\begin{array}{ll}5.3 \text { Structure equations of a lightlike submanifold } & 170\end{array}$

$\begin{array}{ll}5.4 \text { Lightlike surfaces of } \mathbb{R}_{1}^{4} & 177\end{array}$ 
6 CR-lightlike submanifolds of indefinite Kaehler manifolds.

6.1 Indefinite Kaehler manifolds

6.2 Lightlike real hypersurfaces of indefinite Kaehler manifolds

6.3 CR-lightlike submanifolds

7 Lightlike hypersurfaces of Lorentz framed manifolds.

7.1 Lorentz framed manifolds

$\begin{array}{ll}7.2 \text { Induced lightlike framed hypersurfaces } & 216\end{array}$

7.3 Lightlike framed hypersurfaces of decomposable manifolds 223

7.4 Lightlike framed hypersurfaces as Killing horizons 230

8 Lightlike hypersurfaces and electromagnetism.

8.1 Electromagnetism in 4-dimensional Minkowski spacetime 233

8.2 Induced electromagnetic tensor field on lightlike hypersurfaces $\quad 237$

8.3 Geometry of induced electromagnetic tensor fields 246

9 Lightlike hypersurfaces and general relativity.

9.1 Induced Einstein field equations 253

9.2 Lightlike hypersurfaces admitting a conformal vector field 258

9.3 Lightlike hypersurfaces admitting an affine conformal vector field $\quad 264$

9.4 Physical examples of lightlike hypersurfaces 268

$\begin{array}{ll}\text { References. } & 274\end{array}$

$\begin{array}{ll}\text { Author Index. } & 287\end{array}$

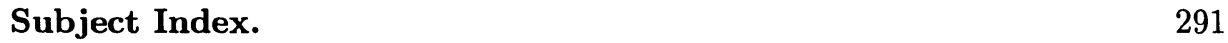




\section{Preface}

This book is about the lightlike (degenerate) geometry of submanifolds needed to fill a gap in the general theory of submanifolds. The growing importance of lightlike hypersurfaces in mathematical physics, in particular their extensive use in relativity, and very limited information available on the general theory of lightlike submanifolds, motivated the present authors, in 1990, to do collaborative research on the subject matter of this book.

Based on a series of author's papers (Bejancu [3], Bejancu-Duggal [1,3], Duggal [13], Duggal-Bejancu [1,2,3]) and several other researchers, this volume was conceived and developed during the Fall '91 and Fall '94 visits of Bejancu to the University of Windsor, Canada.

The primary difference between the lightlike submanifold and that of its nondegenerate counterpart arises due to the fact that in the first case, the normal vector bundle intersects with the tangent bundle of the submanifold. Thus, one fails to use, in the usual way, the theory of non-degenerate submanifolds (cf. Chen [1]) to define the induced geometric objects (such as linear connection, second fundamental form, Gauss and Weingarten equations) on the lightlike submanifold. Some work is known on null hypersurfaces and degenerate submanifolds (see an up-to-date list of references on pages 138 and 140 respectively).

Our approach, in this book, has the following outstanding features: (a) It is the first-ever attempt of an up-to-date information on null curves, lightlike hypersurfaces and submanifolds, consistent with the theory of non-degenerate submanifolds. (b) Our geometric technique is most general, and, has potential for further research on this new topic in differential geometry and other areas of mathematics and physics. (c) We have provided a considerable amount of geometric and physical results on 2 and 3 dimensional lightlike surfaces and hypersurfaces, respectively, of Lorentz manifolds, as an attempt to bring closer mathematicians and physicists.

Chapters 1 and 2 contain most of the prerequisites for reading the rest of the book.

Chapter 3 deals with the fundamental existence and uniqueness theorem of null curves in Lorentz manifolds followed by some results when the ambient manifold is spacetime of general relativity or $n$-dimensional Minkowski space.

Chapter 4 is the core of this book, introducing the most general differential geometric technique to deal with all the induced geometric objects on the lightlike 
hypersurfaces under study. Key result in this chapter is proof of the existence of lightlike hypersurfaces. For physical use, we have provided considerable information on Monge hypersurfaces of Minkowski spaces.

Chapters 5 and 6 are devoted to the general theory of lightlike submanifolds and Cauchy-Riemann (CR) submanifolds respectively. The results of these chapters are primarily based on the present author's papers (Bejancu-Duggal [3] and DuggalBejancu $[1,2,3])$. The background material of Chapter 6 comes from the works of Bejancu [1] and Chen $[1,2,3]$.

Chapters 7, 8 and 9 have been specifically written to apply the theory of lightlike hypersurfaces (cf. chapter 4) to relativity. The background material of these chapters comes from Yano [3], Adler et al. [1], Hawking-Ellis [1], Kramer et al. [1] and Duggal [7,9-12]. It is important to mention that, since this is the first book on lightlike submanifolds, the scope of applications has been limited to interaction with some results on Killing horizon, electromagnetic and radiation fields and asymptotically flat spacetimes. Also, scattered through these chapters there are some new physical results to stimulate interest for further research.

It is our hope that the audience of this book will include graduate students and researchers who have a basic acquaintance with semi-Riemannian geometry and its submanifolds and interest to learn its lightlike counterpart. In general, this book should be appropriate for a two semester graduate course in mathematics and or physics, provided the instructor exercises some selection in the most difficult areas. In particular, for those interested in mathematical physics, the instructor may skip chapters 5 and 6 and with further appropriate selections may cover the rest of the material in one semester. As a reference book, it should be readable to graduate students, research assistants and faculty.

This work was supported through the operating grant of the first author (Duggal), awarded by the Natural Sciences and Engineering Research Council of Canada (NSERC) and the University of Windsor Research Board (UWRB). The second author (Bejancu) wishes to thank the University of Windsor for appointing him visiting professor (Fall '91 and Fall '94) and its Department of Mathematics and Statistics for providing him hospitality and kind support.

Both authors are grateful to all authors of books and articles whose work they have used in preparing this book.

With great pleasure we thank Mrs. Janet Moore for excellent typing of the manuscript. Thanks also go to Dr. Tim Traynor of the Department of Mathematics and Statistics, University of Windsor, for help with $\mathrm{T}_{\mathrm{E}} \mathrm{X}$, including modification of the style file, and to the Departmental secretaries for typing a part of the manuscript. This book was produced using $\mathcal{A M}_{\mathcal{M}} \mathcal{S}-\mathrm{T}_{\mathrm{EX}}$.

Last, but not least, we are grateful to Kluwer Academic Publishers for their support in the preparation of the camera-ready manuscript and excellent care in publishing the volume. 